\title{
Disponibilidade e Valor Nutritivo de Forragem de Leguminosas Nativas (Adesmia DC.) e Exóticas (Lotus L.) ${ }^{1}$
}

\section{Simone Meredith Scheffer-Basso ${ }^{2}$, Aino Victor Avila Jacques ${ }^{3}$, Miguel Dall'Agnol', João Riboldi ${ }^{5}$, Stela Maris Jesuz Castro ${ }^{6}$}

\begin{abstract}
RESUMO - O gênero Adesmia DC. possui 17 espécies nativas no Brasil, distribuídas nos Estados do Sul, cuja importância está vinculada a sua adaptação ao solo e clima regionais, além de ser de crescimento hibernal (temperadas). Este trabalho teve o objetivo comparar o padrão de acúmulo de matéria seca (MS) e valor nutritivo de forragem de A. latifolia, A. punctata e A. tristis, tendo como padrão Lotus corniculatus (cornichão) e L. uliginosus. O ensaio foi realizado em casa de vegetação durante 210 dias (4000 graus-dia). A disponibilidade de forragem (DF) foi similar entre A. latifolia (276 g MS $\left./ \mathrm{m}^{2}\right)$ e cornichão $\left(275 \mathrm{~g} \mathrm{MS} / \mathrm{m}^{2}\right)$ e entre A. tristis (201g MS/m²) e L. uliginosus (192 g MS/m²), sendo que A. punctata apresentou a menor DF (155 g MS/m² $)$. A. latifolia caracterizou-se pela maior precocidade na DF, devido ao crescimento mais rápido em relação às demais espécies, sugerindo seu potencial para utilização durante a estação fria. Em relação às análises de qualidade, o teor de proteína bruta (PB) nas folhas de A. latifolia foi de até $21,6 \%$ e a DIVMO atingiu 72,3\%. Os maiores conteúdos de PB e DIVMO foram encontrados nas folhas de cornichão, 30,3 e 75,8\%, respectivamente. A. tristis apresentou DIVMO muito baixa nos caules (34,9 a 44,7\%), o que poderia limitar seu consumo por bovinos. Concluiu-se que, entre as espécies de Adesmia estudadas, A. latifolia detém o maior potencial forrageiro, sugerindo a continuidade de estudos com a espécie.
\end{abstract}

Palavras-chave: Adesmia, digestibilidade, forragem, leguminosas, Lotus, proteína bruta

\section{Availability and Nutritive Value of the Wild Leguminous (Adesmia DC.) and Exotics (Lotus L.)}

\begin{abstract}
The genus Adesmia DC. has 17 species native to Brazil, distributed in the Southern states, whose importance is linked to its adaptation to the soils and climatic conditions of the region, besides being an active winter-growing species (temperate). This work aimed to compare the patterns of dry matter (DM) accumulation and nutritive value of A. latifolia, A. punctata and A. tristis, using Lotus corniculatus (birdsfoot trefoil) and L. uliginosus (big trefoil), as checks. The experiment was carried out in the greenhouse for 210 days (4000 degrees-day). The forage availability (FA) was similar for A. latifolia $\left(276 \mathrm{~g} \mathrm{DM} / \mathrm{m}^{2}\right)$ and birdsfoot trefoil $\left(275 \mathrm{~g} \mathrm{DM} / \mathrm{m}^{2}\right)$, as well as for A. tristis $\left(201 \mathrm{~g} \mathrm{DM} / \mathrm{m}^{2}\right)$ and big trefoil $\left(192 \mathrm{~g} \mathrm{DM} / \mathrm{m}^{2}\right)$, while A. punctata showed the smallest FD $\left(155 \mathrm{~g} \mathrm{DM} / \mathrm{m}^{2}\right)$. A. latifolia was characterized by an early FA, due to its fast growth when compared to other species, pointing to its potential utilization during the cold season. In relation to the quality analysis, the crude protein $(\mathrm{CP})$ in A. latifolia leaves was up to $21.6 \%$ and the organic matter in vitro digestibility (OMIVD) was up to $72.3 \%$. The highest CP and OMIVD was found in the birdsfoot trefoil leaves, 30.3 and $75.8 \%$, respectively. A. tristis presented a very low OMIVD in the stems, from 34.9 to $44.7 \%$, which could limit its intake by cattle. It is concluded that, among the Adesmia species studied, A. latifolia holds the greatest forage potential and deserves further study.
\end{abstract}

Key Words: Adesmia, Lotus, crude protein, digestibility, forage, leguminous

\section{Introdução}

A atividade pecuária do Rio Grande do Sul depende das pastagens naturais, tipicamente subtropicais, cuja forte estacionalidade de produção gera deficiência de forragem nos meses de outono-inverno. A introdução de leguminosas nesses campos é uma alternativa para minorar tal problema. Todavia, a disponibilidade de sementes em nível comercial recai sobre espécies exó- ticas, exigentes em fertilidade, o que incrementa os custos de produção, em face da necessidade da correção da acidez e fertilidade do solo. Esse fator tem estimulado a busca por espécies nativas, já adaptadas às restrições do solo e que respondam a pequenas quantidades de fertilizantes (HOPKINS et al., 1996; RITTER e SORRENSON, 1985).

Entre as leguminosas nativas hibernais do Sul do Brasil, MORAES e DALL' AGNOL (1990) conside-

\footnotetext{
1 Parte da tese de doutorado do primeiro autor, trabalho financiado por Finep-Pronex

2 Eng ..-Agra., Professora da Universidade de Passo Fundo - Cx. P.611, Cep 99.001-970, Passo Fundo, RS. E.mail: simone@upf.tche.br

3 Engo.-Agro., Ph.D., Pesquisador CNPq, UFRGS - Cx. Postal 776, Porto Alegre, RS, Cep 90012-970. E.mail: aino@vortex.ufrgs.br

${ }^{4}$ Engo.-Agro., Ph.D., Prof. Depto. Plantas Forrageiras e Agrometeorologia, UFRGS. E.mail: migueld@vortex.ufrgs.br

${ }^{5}$ Engo-.Agro., Dr., Prof., Depto. Estatística, UFRGS.

${ }^{6}$ Estatístisca, MSc., Prof., Depto. Estatística, UFRGS.
} 
ram importantes os gêneros Adesmia DC., Lathyrus L., Ornithopus L., Trifolium L. e Vicia L. As observações a campo de Adesmia indicam que suas espécies são consumidas pelo gado e não há registro de substâncias tóxicas (BURKART, 1952). O comportamento sob pastejo, porém, não está esclarecido, havendo informações contraditórias. ALLEN e ALLEN (1981) mencionam forte vitalidade após pastejo contínuo, enquanto VALLS (1984) considera essas espécies como pouco resistentes a altas pressões de pastejo, com o que a maioria tende a desaparecer. No Brasil, o interesse pela coleta e pela conservação de germoplasma de Adesmia, de forma sistemática, iniciou-se a partir de 1970, devendo-se, segundo MIOTTO e LEITÃO FILHO (1993), ao fato de várias espécies do gênero serem potencialmente boas forrageiras para as regiões de clima temperado, com estações bem limitadas, invernos frios e ocorrência de geadas. Nessas condições, tais plantas têm sido encontradas vegetando e permanecendo verdes quando os campos estão secos e quase totalmente desprovidos de vegetação. Entre as 17 espécies nativas do Sul do Brasil estão A. latifolia (Spreng.) Vog. (estolonífera), A. punctata (Poir.) DC. (estolonífera, ereto-decumbente) e A. tristis Vog. (subarbustiva, ereta), sendo que a primeira se destaca pela exuberância de suas folhas, as maiores do gênero. Considerando-se as leguminosas exóticas, que tem sido utilizadas no melhoramento das pastagens naturais, destaca-se o gênero Lotus L., pela maior tolerância à acidez em relação aos trevos (Trifolium spp.). O cornichão (L. corniculatus L.) teve sua história no Rio Grande do Sul iniciada em 1940, quando foi introduzido, sendo que a partir de 1955 iniciaram os trabalhos de melhoramento, culminando com o lançamento da cv. São Gabriel, cerca de quinze anos mais tarde (PAIM, 1988). Tanto no Rio Grande do Sul, desde que foi introduzido, quanto no Uruguai, a popularidade do cornichão está alicerçada no seu alto valor nutritivo, na sua pouca exigência quanto às condições do solo e no fato de não causar timpanismo (LÓPEZ et al., 1966; FORMOSO, 1993). No caso de L. uliginosus Schkuhr, os trabalhos desenvolvidos têm mostrado a necessidade de seleção de material mais adequado às condições do Rio Grande do Sul, onde seu uso tem sido restringido pelo lento estabelecimento e pela baixa produção e difícil colheita de sementes. Tal característica parece estar aliada ao esparso ou, até mesmo, à ausência de florescimento no ano do estabelecimento (PAIM e RIBOLDI, 1991).
Este trabalho foi desenvolvido com o objetivo de avaliar, sob condições de casa de vegetação, a dinâmica de acúmulo e valor nutritivo de forragem de $A$. latifolia, A. punctata, A. tristis, tendo como padrão L. corniculatus e L. uliginosus, com a finalidade sugerir novos trabalhos e promover uma triagem para futuros trabalhos de coleta e caracterização de germoplasma de Adesmia DC.

\section{Material e Métodos}

O experimento foi conduzido em casa de vegetação, na Universidade de Passo Fundo, Rio Grande do Sul, com coordenadas $28^{\circ} 15^{\prime} \mathrm{S}$ e $52^{\circ} 24^{\prime} \mathrm{W}$ e $687 \mathrm{~m}$ de altitude média. $O$ ensaio constituiu-se num bifatorial, sendo que os tratamentos consistiram de sete leguminosas (fator leguminosa) amostradas em sete épocas (fator época), organizados em delineamento de blocos casualizados, com quatro repetições. Foram testados dois acessos de Adesmia latifolia (BRA-001422, A. latifolia 1, BRA-001465, A. latifolia 2), dois de Adesmia tristis (BRA-001449, A. tristis 1, BRA-001457, A.tristis 2), um de Adesmia punctata (BRA-001431), um cultivar de L. corniculatus (cv. São Gabriel) e um cultivar de L. uliginosus (cv. Grassland Maku). As unidades experimentais consistiram de caixas de madeira, forradas com lona plástica perfurada na base, medindo $0,89 \mathrm{~m} \times 1,00 \mathrm{~m}$ e $0,20 \mathrm{~m}$ de profundidade. O solo utilizado, coletado de uma área de pastagem natural, pertence à unidade de mapeamento Passo Fundo, sendo um latossolo vermelho-escuro distrófico, com textura argilosa, tendo basalto como substrato. A análise química revelou: $61,70 \%$ de argila, $\mathrm{pH}$ água $=4,30, \mathrm{pH} \mathrm{SMP}=4,60, \mathrm{P}=2,0 \mathrm{mg} / \mathrm{L}, \mathrm{K}=26 \mathrm{mg} / \mathrm{L}$, $\mathrm{M} . \mathrm{O}=3,9 \%, \mathrm{Al}=4,3 \mathrm{cmol} / \mathrm{L}, \mathrm{Ca}=1,2 \mathrm{cmol} / \mathrm{L}$, $\mathrm{Mg}=0,9 \mathrm{cmol} / \mathrm{L}, \mathrm{S}=2,2 \mathrm{cmol} / \mathrm{L}, \mathrm{CTC}=14,7 \mathrm{cmol} / \mathrm{L}$, $\mathrm{V}=14,7 \%$ e saturação de $\mathrm{Al}=69,1 \%$. Cada caixa recebeu cerca de $160 \mathrm{~kg}$ de solo seco ao ar, corrigido com o equivalente a 12 t/ha de calcário, dose recomendada para elevar o pH a 6,0. Após a aplicação de calcário, três meses antes do plantio, o pH (água) estava em 5,1 e o pH SMP, em 6,0. Na adubação, foram aplicados $390 \mathrm{~kg} / \mathrm{ha}$ de $\mathrm{P}_{2} \mathrm{O}_{5}$ e $270 \mathrm{~kg} / \mathrm{ha}$ de $\mathrm{K}_{2} \mathrm{O}$, quantidades que representaram o triplo da recomendação constante das normas oficiais (SOCIEDADE BRASILEIRA DE CIÊNCIA DE SOLO, 1997) para leguminosas de estação fria. Como fontes de fósforo e potássio foram utilizados o superfostato triplo e o cloreto de potássio, que foram incorporados ao solo 55 dias antes do plantio. Em seguida, foi feita 
uma irrigação até o ponto de escorrimento com intuito de uniformizar a umidade do solo nas unidades experimentais, que, em seguida, foram cobertas com lâminas de isopor. A adubação com enxofre e micronutrientes foi realizada quinze dias antes do plantio, nas seguintes quantidades: $\mathrm{S}=5,0 \mathrm{mg} / \mathrm{kg}$ de solo, na forma de $\mathrm{MgSO}_{4} \cdot 7 \mathrm{H}_{2} \mathrm{O}, \mathrm{B}=0,5 \mathrm{mg} / \mathrm{kg}$ de solo, na forma de $\mathrm{H}_{3} \mathrm{BO}_{3}, \mathrm{Cu}=1,0 \mathrm{mg} / \mathrm{kg}$ de solo, como $\mathrm{CuSO}_{4} \cdot 7 \mathrm{H}_{2} \mathrm{O}, \mathrm{Mo}=0,1 \mathrm{mg} / \mathrm{kg}$ de solo, na forma de $\left(\mathrm{NH}_{4}\right) 6 \mathrm{Mo}_{7} \mathrm{O}_{24} \cdot 4 \mathrm{H}_{2}$ e $\mathrm{Zn}=1,0 \mathrm{mg} / \mathrm{kg}$ de solo, na forma de $\mathrm{ZnSO}_{4} \cdot 7 \mathrm{H}_{2} \mathrm{O}$. No dia do plantio foram retiradas amostras de solo para análise, na qual se constatou: $\mathrm{P}=37,0 \mathrm{mg} / \mathrm{L}, \mathrm{K}=200,0 \mathrm{mg} / \mathrm{L}$, $\mathrm{Al}=0,0 \mathrm{cmol} / \mathrm{L}, \mathrm{Ca}=8,1 \mathrm{cmol} / \mathrm{L}, \mathrm{Mg}=2,0 \mathrm{cmol} / \mathrm{L}$, $\mathrm{S}=10,6 \mathrm{cmol} / \mathrm{L}, \mathrm{CTC}=12,4 \mathrm{cmol} / \mathrm{L}, \mathrm{V}=85,9 \% \mathrm{e}$ saturação de $\mathrm{Al}=0,0 \%$. Como tratamento fungicida preventivo, foi aplicada, quinze dias antes do plantio, uma mistura de quintozene (75\% PM) a $300 \mathrm{~g} / 100 \mathrm{~L}$ de água, metalaxil (35\% PM) a $200 \mathrm{~g} / 100 \mathrm{~L}$ de água e benomil (50\% PM) a $70 \mathrm{~g} / 100 \mathrm{~L}$ de água, na quantidade de dois litros da calda $/ \mathrm{m}^{2}$ de solo; onze dias após o plantio, foi feita uma segunda aplicação com uma mistura de benomil a $0,05 \%$ e quintozene a 0,3\% (REIS e FORCELINI, 1994). Para a obtenção das plântulas, as sementes de Adesmia foram previamente escarificadas em água a $100^{\circ} \mathrm{C}$, durante cinco minutos; as sementes de Lotus não foram escarificadas. Todas as sementes ficaram em germinador a $25^{\circ} \mathrm{C}$ durante cinco dias, sendo que em 30 de abril de 1996 foram transferidas para as unidades experimentais, obtendo-se população média de
100 plantas $/ \mathrm{m}^{2}$, dispostas em linhas distantes $0,15 \mathrm{~m}$. Como as plântulas de Lotus não sobreviveram ao transplante, foi realizada a semeadura direta no solo em 25 de maio. As sementes de Adesmia e Lotus não foram inoculadas, por não se dispor de rizóbio selecionada para as primeiras. Assim, durante o experimento, o nitrogênio foi suprido somente na forma de uréia, na dose de $80 \mathrm{~kg} \mathrm{~N} / \mathrm{ha}$, fracionados em quatro aplicações, para todas as leguminosas. Foi adotada a soma térmica (ST) ou seja, o acúmulo de graus-dia (GD), para cálculo do período de crescimento, de acordo com ROMO e EDDLEMAN (1995), considerando-se a temperatura basal de zero grau $\left(0^{\circ} \mathrm{C}\right)$ para ambos os gêneros. Para isso, foram tomadas diariamente as temperaturas mínimas e máximas ocorridas dentro da casa de vegetação.

A retirada das amostras em cada época de amostragem foi realizada da seguinte maneira: os blocos foram amostrados dois a dois, de forma que, após o término das análises laboratoriais das plantas amostradas dos primeiros blocos, era realizada a amostragem nos outros dois. Isso explica as diferenças na soma térmica e no número de dias apresentadas na Tabela 1.

A área amostral foi de $0,20 \mathrm{~m} \times 0,10 \mathrm{~m}$ nas quatro primeiras avaliações e de $0,20 \mathrm{~m}$ x $0,30 \mathrm{~m}$ nas três últimas. As plantas foram retiradas e separadas para fins de pesagem em raízes, folhas ativas, folhas senescentes, hastes vegetativas e hastes floríferas. Portanto, a matéria seca (MS) acumulada no nível da superfície do solo era composta de folhas e hastes. O valor nutritivo foi avaliado por intermédio do teor de

Tabela 1 - Épocas de avaliação, acúmulo de graus-dia (GD) e número de dias após o plantio (d.a.p.) correspondente ao período de crescimento de Adesmia e Lotus

Table 1 - Sampling date, cumulative degree-days (DD) and number of days after sowing (d.a.s) corresponding to the growing period of Adesmia and Lotus

\begin{tabular}{|c|c|c|c|c|c|}
\hline \multirow{2}{*}{$\begin{array}{l}\text { Épocas de avaliação } \\
\text { Evaluation time }\end{array}$} & \multirow{2}{*}{$\begin{array}{c}\text { Blocos } \\
\text { Blocks }\end{array}$} & \multicolumn{2}{|c|}{ Lotus } & \multicolumn{2}{|c|}{ Adesmia } \\
\hline & & $\begin{array}{l}\mathrm{GD}\left({ }^{\circ} \mathrm{C}\right) \\
D D\left({ }^{\circ} \mathrm{C}\right)\end{array}$ & $\begin{array}{l}\text { d.a.p. } \\
\text { d. a. s. }\end{array}$ & $\begin{array}{l}\mathrm{GD}\left({ }^{\circ} \mathrm{C}\right) \\
D D\left({ }^{\circ} \mathrm{C}\right)\end{array}$ & $\begin{array}{l}\text { d.a.p. } \\
\text { d.a.s. }\end{array}$ \\
\hline \multirow[t]{2}{*}{25 a 29/7/96 } & I e II & 1535 & 86 & 952 & 56 \\
\hline & III e IV & 1794 & 90 & 1122 & 60 \\
\hline \multirow[t]{2}{*}{09 a 15/8/96 } & I e II & 1829 & 101 & 1246 & 71 \\
\hline & III e IV & 2132 & 107 & 1458 & 77 \\
\hline \multirow[t]{2}{*}{26 a $30 / 8 / 96$} & I e II & 2160 & 118 & 1577 & 88 \\
\hline & III e IV & 2414 & 122 & 1740 & 92 \\
\hline \multirow[t]{2}{*}{12 a $17 / 9 / 96$} & I e II & 2421 & 135 & 1839 & 105 \\
\hline & III e IV & 2726 & 140 & 2052 & 110 \\
\hline \multirow{2}{*}{ 27/9 a 04/10/96 } & I e II & 2732 & 150 & 2150 & 120 \\
\hline & III e IV & 3072 & 157 & 2398 & 127 \\
\hline \multirow[t]{2}{*}{ 25/10 a 01/11/96 } & I e II & 3307 & 178 & 2725 & 148 \\
\hline & III e IV & 3653 & 185 & 2980 & 155 \\
\hline \multirow[t]{2}{*}{22 a $29 / 11 / 96$} & I e II & 3991 & 206 & 3409 & 176 \\
\hline & III e IV & 4328 & 213 & 3654 & 183 \\
\hline
\end{tabular}


proteína bruta $(\mathrm{PB})$ e digestibilidade in vitro da matéria orgânica (DIVMO), sendo as amostras de folhas e caules reunidas de acordo com o estádio fenológico dos acessos e cultivares em vegetativo, pré-florescimento e florescimento pleno. Assim, independentemente do acúmulo de graus-dia, para o valor nutritivo foram considerados os estádios de desenvolvimento, devidamente determinados ao longo do período experimental. Para a fração folha, foram misturadas as folhas em senescência e as folhas ativas e, para a fração caule, foram incluídas todas as hastes. A porcentagem de PB foi obtida pela multiplicação do teor de $\mathrm{N}$ por 6,25 , determinado pelo método Micro-Kjeldhal, e a DIVMO foi feita segundo o método de TILLEY e TERRY (1963). Os dados relativos à análise de crescimento foram analisados por intermédio da análise de variância multivariada para dados longitudinais e análise de regressão para graus-dia. Para PB foi efetuada a análise da variância e as médias, comparadas por DMS a 5\%. Os resultados da DIVMO não foram analisados estatisticamente, devido à insuficiência de amostras para alguns tratamentos.

\section{Resultados e Discussão}

$\mathrm{Na}$ análise da variância observou-se efeito $(\mathrm{P}<0,05)$ da interação Leguminosa $\mathrm{x}$ Época para $\mathrm{o}$ acúmulo de MS, cujo comportamento em função de graus-dia foi analisado mediante análise de regressão (Figura 1).

Apesar de as espécies de Lotus terem acumulado menor soma térmica, em função da necessidade de nova semeadura, 30 dias após o primeiro plantio, elas apresentaram acúmulos de MS equivalentes ou superiores às de Adesmia. Enquanto o cornichão foi um bom padrão para A. latifolia, o L. uliginosus o foi para as demais espécies de Adesmia. Assim, verificou-se que os maiores acúmulos de MS foram apresentados por A. latifolia 2 e cornichão, indicando disponibilidade de forragem de $280 \mathrm{~g} \mathrm{MS} / \mathrm{m}^{2}$ (Figura 1). Dados similares foram reportados por DUTRA et al. (1998a), que, ao introduzirem A. latifolia em campo nativo, obtiveram o equivalente a $250 \mathrm{~g} \mathrm{MS} / \mathrm{m}^{2}$. Esses resultados também são comparáveis às produções de trevo-branco (Trifolium repens $\mathrm{L}$.) verificadas por CHAPMAN e CARADUS (1997), de 92,2 a $271 \mathrm{~g}$ $\mathrm{MS} / \mathrm{m}^{2}$, e por BRINCK et al. (1992), de $250 \mathrm{~g} \mathrm{MS} / \mathrm{m}^{2}$. A semelhança entre esses resultados é estimulante, considerando-se que A. latifolia obteve tais rendimentos em apenas 3000 GD (150 dias) e nos meses seguintes à semeadura. Com o cornichão, BULLARD

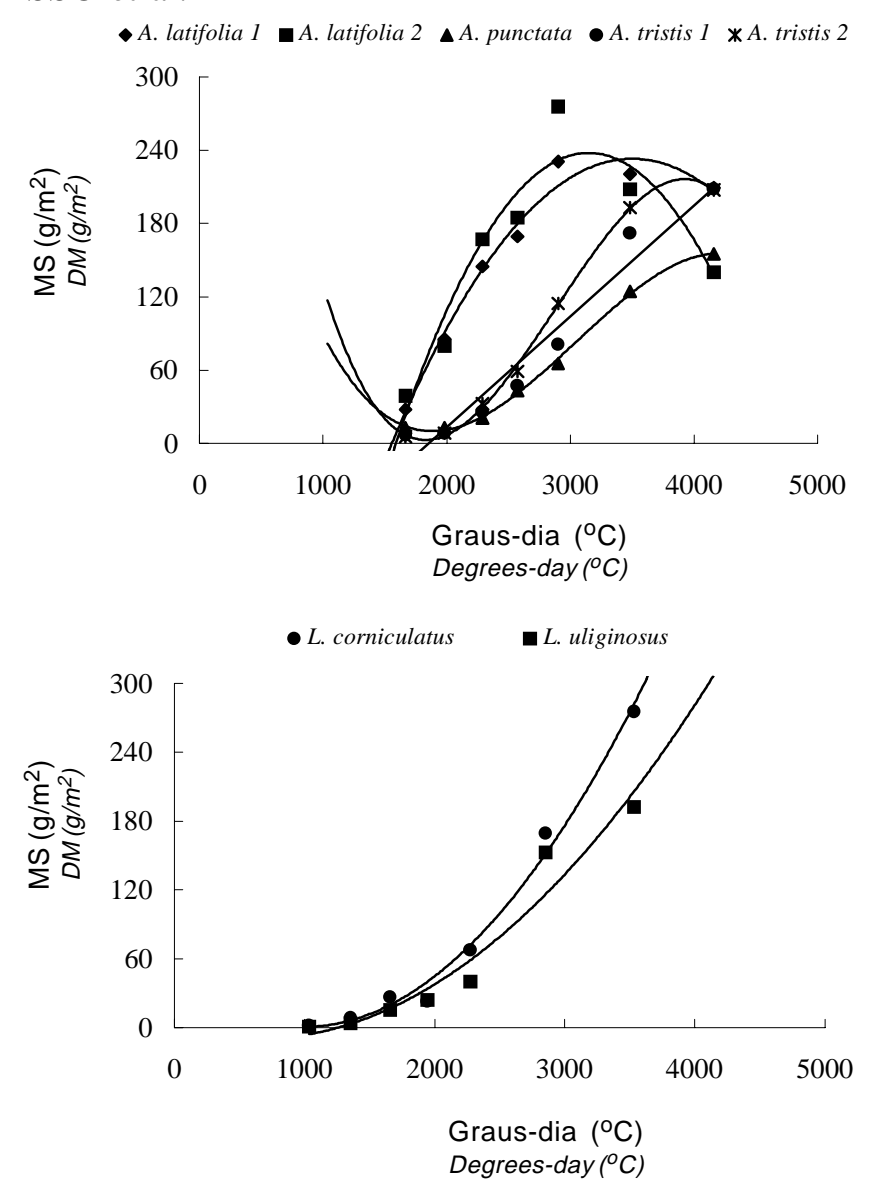

\begin{tabular}{llc} 
Leguminosas & \multicolumn{1}{c}{ Equação } & $\mathrm{R}^{2}$ \\
\hline A. latifolia 1 & $\mathrm{y}=-506,74+0,41 \mathrm{x}-0,000058 \mathrm{x}^{2}$ & 0,81 \\
A. latifolia 2 & $\mathrm{y}=-729,38+0,61 \mathrm{x}-0,000098 \mathrm{x}^{2}$ & 0,68 \\
A. punctata & $\mathrm{y}=372-0,45 \mathrm{x}+0,0002 \mathrm{x}^{2}-1,8 \mathrm{E}-8 \mathrm{x}^{3}$ & 0,89 \\
A. tristis 1 & $\mathrm{y}=-169,56+0,091 \mathrm{x}$ & 0,80 \\
A. tristis 2 & $\mathrm{y}=465,13-0,635 \mathrm{x}+0,00026 \mathrm{x}^{2}-3 \mathrm{E}-8 \mathrm{x}^{3}$ & 0,98 \\
L. corniculatus & $\mathrm{y}=41,67-0,085 \mathrm{x}+0,0004 \mathrm{x}^{2}$ & 0,98 \\
L. uliginosus & $\mathrm{y}=39,17-0,077 \mathrm{x}-0,000037 \mathrm{x}^{2}$ & 0,88 \\
\hline
\end{tabular}

Figura 1 - Acúmulo de matéria seca (MS) da parte aérea de Adesmia e Lotus, em função de graus-dia.

Figure 1 - Shoot dry matter accumulation (DM) of Adesmia and Lotus as a function of the degree-days.

e CRAWFORD (1995) obtiveram entre 386 e $934 \mathrm{~g}$ $\mathrm{MS} / \mathrm{m}^{2}$, porém com estandes de 14 meses de idade, ao passo que, neste estudo, a MS foi acumulada em apenas 150 dias. McGRAW et al. (1986) estimaram produções de forragem de 473 a $530 \mathrm{~g} \mathrm{MS} / \mathrm{m}^{2}$ nessa espécie no início do florescimento. Nas outras leguminosas, o acúmulo de MS aérea foi de, aproximadamente, $160 \mathrm{~g} \mathrm{MS} / \mathrm{m}^{2}$ para A. punctata e de 150 a $200 \mathrm{~g} \mathrm{MS} / \mathrm{m}^{2}$, respectivamente, para L. uliginosus e A. tristis. Ressalta-se que a redução da disponibilidade de MS de A. latifolia (Figura 1) pode indicar um período mais restrito de acúmulo de 
forragem em relação às demais. Essa espécie estabilizou o acúmulo de MS próximo aos $3000 \mathrm{GD}$, ao passo que, em A. punctata e A. tristis, isso somente ocorreu aos $4000 \mathrm{GD}$ de crescimento, denotando nessas últimas um ritmo mais lento de crescimento.

Entre as espécies mais produtivas, A. latifolia $\mathrm{e}$ cornichão, é importante destacar a diferença na estacionalidade do acúmulo de MS: A. latifolia alcançou seu patamar produtivo aos $2.902 \mathrm{GD}$, enquanto o cornichão mostrou-se mais tardio, precisando acumular um adicional de 630 GD para a mesma disponibilidade de forragem $\left(280 \mathrm{~g} \mathrm{MS} / \mathrm{m}^{2}\right)$. É relevante a maior precocidade de A. latifolia em relação ao cornichão, o que não é comum em espécies estoloníferas. Por se tratar de espécie nativa, ainda não sujeita a trabalhos de melhoramento genético, seu comportamento e hábito de crescimento a habilitam para programas de pesquisa na área. É importante destacar que a disponibilidade de forragem no início de período frio é uma característica altamente desejável e perseguida pelos pesquisadores. Para cornichão, está bem estabelecido seu pico de produção a partir do final do inverno. Por essa razão é considerada uma leguminosa temperada de crescimento estival, o que dificulta seu estabelecimento entre as gramíneas subtropicais das pastagens naturais (SANTIÑAQUE e CARÁMBULA 1981).

Além das diferenças estacionais, outro aspecto importante, em termos de produção animal, diz respeito à composição da forragem. Na Tabela 2, na qual estão expressos os valores de relação folha:caule
(RFC) das leguminosas, verifica-se que as espécies com maior RFC foram as estoloníferas, A. latifolia e A. punctata, em relação às eretas (A. tristis e cornichão). HAYCOCK (1984) observou valores similares em trevo-branco, com RFC de 4,8 durante o inverno. Se for considerada uma ST próxima de $2900 \mathrm{GD}$, para fins de equiparação do tempo de crescimento das espécies, verifica-se, na Tabela 2, que A. tristis apresentou RFC superior ao cornichão. A partir daí houve declínio expressivo na RFC dessa espécie, ao passo que no cornichão ocorreu uma maior estabilidade. Esse comportamento demonstra uma das vantagens do hábito indeterminado dessa espécie, sendo um dos fatores de sua reconhecida qualidade. A constante formação de hastes vegetativas é uma das causas de declínio mais lento da qualidade da forragem do cornichão; por essa razão, o diferimento e o atraso na colheita têm sido práticas de manejo sugeridas pelos pesquisadores (BEUSELINCK et al., 1984).

A análise de variância mostrou efeito $(\mathrm{P}<0,05)$ de leguminosa e de fração (caule, folha) sobre o teor de PB. Independentemente de estádio fenológico, a fração folha apresentou, na média geral, os maiores teores de $\mathrm{PB}$, atingindo 20,98\% em relação à fração caule, com 10,34\% (Tabela 3).

Considerando-se as variações ocorridas ao longo do desenvolvimento das plantas, observa-se que houve decréscimo em caules e folhas, com maior redução nas folhas. Isso foi constatado por LÓPEZ et al. (1966), em cornichão, no qual o teor de PB nas folhas diminuiu de um valor máximo de 29,81 para 20,98\%,

Tabela 2 - Relação folha:caule (RFC) de Adesmia e Lotus durante o período de crescimento Table 2 - Leaf:stem ratio (LSR) of Adesmia and Lotus during the growth period

\begin{tabular}{lr}
\hline Leguminosas & $\operatorname{RFC}\left(\mathrm{g} \mathrm{g}^{-1}\right)$ \\
Leguminous & $\operatorname{LSR}\left(g g^{-1}\right)$
\end{tabular}

\begin{tabular}{|c|c|c|c|c|c|c|c|c|}
\hline & \multicolumn{7}{|c|}{$\begin{array}{c}\text { Graus-dia }\left({ }^{\circ} \mathrm{C}\right) \\
\text { Degrees-day }\left({ }^{\circ} \mathrm{C}\right)\end{array}$} & \multirow[t]{2}{*}{$\begin{array}{c}\text { Média } \\
\text { Mean }\end{array}$} \\
\hline & 1665 & 1981 & 2287 & 2574 & 2902 & 3480 & 4160 & \\
\hline A. latifolia 1 & 4,83 & 4,09 & 3,34 & 3,83 & 2,32 & 0,91 & 0,38 & 2,81 \\
\hline A. latifolia 2 & 4,10 & 2,85 & 5,90 & 2,87 & 3,26 & 1,71 & 0,90 & 3,08 \\
\hline A. punctata & 11,35 & 11,44 & 5,73 & 3,17 & 5,27 & 1,32 & 0,62 & 5,56 \\
\hline A. tristis 1 & 3,88 & 3,57 & 2,58 & 1,68 & 1,56 & 0,47 & 0,24 & 2,00 \\
\hline \multirow[t]{4}{*}{ A. tristis 2} & 5,28 & 4,25 & 1,83 & 1,57 & 0,88 & 0,29 & 0,27 & 2,05 \\
\hline & \multirow{2}{*}{\multicolumn{7}{|c|}{$\begin{array}{c}\text { Graus-dia }\left({ }^{\circ} \mathrm{C}\right) \\
\text { Degrees-day }\left({ }^{\circ} \mathrm{C}\right)\end{array}$}} & \multirow{3}{*}{$\begin{array}{l}\text { Média } \\
\text { Mean }\end{array}$} \\
\hline & & & & & & & & \\
\hline & 1037 & 1352 & 1659 & 1946 & 2274 & 2853 & 3532 & \\
\hline L. corniculatus & 1,88 & 5,03 & 2,34 & 2,04 & 1,33 & 0,60 & 0,53 & 1,96 \\
\hline L. uliginosus ${ }^{1}$ & 5,50 & 6,23 & 1,63 & 1,33 & 1,04 & 0,38 & 0,33 & 2,35 \\
\hline
\end{tabular}

${ }^{1}$ Não estão incluídos os rizomas na fração caule.

${ }^{1}$ Rhizomes are not included in the stem fraction portion. 
enquanto, para caule, houve redução de 12,46 para 9,97\% nos estádios vegetativo e de formação de sementes, respectivamente. Considerando-se as diferenças interespecíficas, as maiores variações foram constatadas nas folhas. Lotus spp. destacaram-se pelo elevado teor de PB nas folhas, similares aos obtidos por DUELL e GAUSMAN (1957) e CAROSO et al. (1982), enquanto A. latifolia apresentou os menores teores. DALL'AGNOL e GOMES (1994) relataram, para A. latifolia, A. punctata e A. tristis, 18,6, 23,4 e 17,2\% de PB no estádio de pré-florescimento. Já COELHO (1996) obteve entre 10,24 e $11,44 \%$ de PB em A. latifolia, 10,01\% em A. tristis e $14,49 \%$ em A. punctata, no início do florescimento. Observa-se, portanto, que, em todos os trabalhos citados, A. latifolia e A. tristis apresentaram os menores teores de PB. No entanto, mesmo com essa aparente inferioridade em relação a Lotus spp., tais valores são muito bons. Fatores como a morfologia e a senescência foliar devem ser considerados na discussão das diferenças entre os gêneros estudados neste trabalho. A. latifolia tem folha composta pinada, de maior comprimento (até $20 \mathrm{~cm}$ ) e, portanto, com substancial proporção de tecido vegetal da ráquis, em relação ao Lotus spp., cujas folhas e folíolos são de pequenas dimensões. A. latifolia também apresentou maior senescência foliar (60\%), ao passo que, no cornichão, permaneceu em $10 \%$.
Esse fator deve ter sido a principal causa da maior redução de $\mathrm{PB}$ em A. latifolia 1 (12 pontos percentuais nas folhas) entre o estádio vegetativo e o florescimento pleno; nas demais leguminosas, tal redução foi de, aproximadamente, oito pontos. $\mathrm{O}$ mesmo vale para a fração caule, já que, para A. latifolia, os estolões senescentes também participaram da amostra. Pelo exposto, ficou nítido que as espécies de Adesmia avaliadas no presente estudo podem ser utilizadas como fonte de proteína, visto manterem teores acima de 7\%, que é o mínimo necessário aos animais ruminantes.

Os dados de DIVMO para folha e caule estão citados na Tabela 4, de acordo com o estádio fenológico. No geral, com o avanço do ciclo de crescimento, houve redução na DIVMO, mais acentuada para caules em relação às folhas. Ao contrário do que foi observado para $\mathrm{PB}$, as variações foram mais marcantes em caule, tanto em relação ao estádio de desenvolvimento quanto às espécies. Para caules, houve diminuição de sete pontos percentuais na fração caule, entre o estádio vegetativo e o florescimento pleno. Em geral, a redução na DIVMS é mais acentuada em caule do que em folhas, pois os caules têm maior quantidade de lignina, que protege parte da celulose e hemicelulose da digestão ruminal (HACKER e MINSON, 1981). A literatura é muito clara quanto aos elevados percentuais de DIVMO das folhas de leguminosas, cujos folíolos são compos-

Tabela 3 - Teor de proteína bruta (PB) das frações caule e folha de Adesmia e Lotus nos estádios vegetativo (V), início de florescimento (IF) e florescimento pleno (FP)

Table 3 - Crude protein content (CP) of stem and leaf fractions of Adesmia and Lotus in the vegetative (V), early flowering (EF) and full flowering (FF) stages

\begin{tabular}{|c|c|c|c|c|c|c|}
\hline \multirow[t]{3}{*}{$\begin{array}{l}\text { Leguminosas } \\
\text { Leguminous }\end{array}$} & \multicolumn{3}{|c|}{$\begin{array}{l}\text { Caule } \\
\text { Stem }\end{array}$} & \multicolumn{3}{|c|}{$\begin{array}{c}\text { Folha } \\
\text { Leaf }\end{array}$} \\
\hline & V & IF & FP & $\mathrm{V}$ & $\mathrm{IF}$ & $\mathrm{FP}$ \\
\hline & $V$ & $E F$ & $F F$ & $V$ & $E F$ & $F F$ \\
\hline & \multicolumn{6}{|c|}{$\begin{array}{c}\text { CP }(\%) \\
C P(\%)\end{array}$} \\
\hline A. latifolia 1 & $12,76^{\mathrm{bc}}$ & $8,28^{\mathrm{b}}$ & $7,24^{\mathrm{b}}$ & $23,47^{\mathrm{c}}$ & $13,11^{\mathrm{c}}$ & $11,72^{\mathrm{c}}$ \\
\hline A. latifolia 2 & $13,06^{\mathrm{bc}}$ & $9,24^{\mathrm{b}}$ & & $21,59^{d}$ & $14,88^{\mathrm{c}}$ & \\
\hline A. punctata & $13,44^{\mathrm{ab}}$ & $9,17 \mathrm{a}$ & $/ 1$ & $23,89^{c}$ & $19,44^{\mathrm{b}}$ & $/ 1$ \\
\hline A. tristis 1 & $11,63^{\mathrm{c}}$ & $9,86^{\mathrm{ab}}$ & $8,87^{\mathrm{a}}$ & $26,64^{\mathrm{b}}$ & $21,82^{b}$ & $18,71^{\mathrm{b}}$ \\
\hline A. tristis 2 & $11,66^{\mathrm{c}}$ & $12,22^{\mathrm{a}}$ & $7,34^{\mathrm{b}}$ & $27,72^{b}$ & $19,08^{b}$ & $19,20^{b}$ \\
\hline L. corniculatus & $14,81^{\mathrm{a}}$ & $11,07^{\mathrm{ab}}$ & $8,87^{\mathrm{a}}$ & $30,34^{\mathrm{a}}$ & $26,11^{\mathrm{a}}$ & $22,92^{\mathrm{a}}$ \\
\hline L. uliginosus & $13,47^{\mathrm{a}}$ & $/ 1$ & $/ 1$ & $29,44^{\mathrm{a}}$ & & \\
\hline Média (estádios) & 12,98 & 9,97 & 8,08 & 25,73 & 19,07 & 18,14 \\
\hline \multicolumn{7}{|l|}{ Mean (stages) } \\
\hline Média (frações) & & 10,34 & & & 20,98 & \\
\hline Mean (fractions) & & & & & & \\
\hline
\end{tabular}

\footnotetext{
Médias seguidas por letras distintas, nas colunas, diferem $(P<0,05)$ pelo teste DMS.

${ }^{1}$ As espécies não atingiram os estádios fenológicos referidos.

Means followed by different letters, within column, differ $(P<0.05)$ by MDS test.

${ }^{1}$ The species did not reach the stages related.
} 
tos de tecidos (mesófilo) rapidamente digeríveis, ainda que algumas espécies apresentem componentes antiqualitativos, especialmente taninos, que reduzem a sua digestibilidade (AKIN, 1989). Pouco se sabe, porém, sobre a digestibilidade do pecíolo das leguminosas (HACKER e MINSON, 1981).

Com relação às diferenças intergenotípicas, A. tristis destacou-se pelos baixos valores, mesmo no estádio vegetativo. Isso não surpreende, dado ao seu hábito subarbustivo, com caules rígidos, sugerindo maior quantidade proporcional de tecido de condução, que, segundo AKIN (1989), não é degradado. $\mathrm{Na}$ fração folhas, as diferenças entre as espécies praticamente inexistiram, com exceção do L. uliginosus. (Tabela 4). A. latifolia foi a espécie do gênero a apresentar os mais elevados percentuais de DIVMO, quando em estádio vegetativo. Os dados obtidos são similares aos de MORAES et al. (1989) em Trifolium riograndense Burk. (DIVMO $=65$ a 69\%), superiores aos encontrados em Trifolium polypmorphum $\mathrm{L}$. (DIVMO $=50,76 \%$ ), mas inferiores ao trevo-branco $(72,36$ a $82,26 \%$ na parte aérea). DALL' AGNOL e GOMES (1994) encontraram entre 70,8 e $78,1 \%$ de DIVMO para A. latifolia nos estádios de florescimento e vegetativo, respectivamente; para A. punctata e A. tristis, em préflorescimento, os valores foram estimados em 62,7 e $64,5 \%$, respectivamente. COELHO (1996) obteve valores de DIVMO de até $81,06 \%$ em A. latifolia, $57,04 \%$ em A. punctata e $57,82 \%$ em A. tristis, todas em pré-florescimento, observando, inclusive, variação intragenotípica na primeira espécie.

Para o cornichão, trabalhos realizados no Rio Grande do Sul citam DIVMO de 57 a $72 \%$ na parte

Tabela 4 - Digestibilidade in vitro da matéria orgânica (DIVMO) das frações caule e folha de Adesmia sp. e Lotus sp. nos estádios vegetativo (V), início de florescimento (IF) e florescimento pleno (FP)

Table 4 - In vitro organic matter (IVOMD) of stem and leaf fractions of Adesmia spp. and Lotus spp. in the vegetative (V), early flowering (EF) and full blooming $(F B)$ stages

\begin{tabular}{|c|c|c|c|c|c|c|}
\hline \multirow[t]{3}{*}{$\begin{array}{l}\text { Leguminosas } \\
\text { Leguminous }\end{array}$} & \multicolumn{3}{|c|}{$\begin{array}{l}\text { Caule } \\
\text { Stem }\end{array}$} & \multicolumn{3}{|c|}{$\begin{array}{l}\text { Folha } \\
\text { Leaf }\end{array}$} \\
\hline & $\mathrm{V}$ & IF & FP & V & IF & $\mathrm{FP}$ \\
\hline & $V$ & $E F$ & $F F$ & $V$ & $E F$ & $F F$ \\
\hline & & & , & )) - & 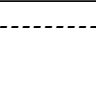 & - \\
\hline A. latifolia 1 & 59,45 & 57,05 & 55,70 & 71,33 & 72,28 & 71,50 \\
\hline A. latifolia 2 & 60,60 & 50,33 & $/ 1$ & 71,85 & 71,10 & $/ 1$ \\
\hline A. punctata & 59,00 & 47,58 & $/ 1$ & 72,45 & 67,98 & $/ 1$ \\
\hline A. tristis 1 & 40,98 & 40,58 & 35,40 & 71,98 & 66,70 & 67,80 \\
\hline A. tristis 2 & 44,73 & 36,50 & 34,85 & 69,15 & 75,05 & 71,30 \\
\hline L. corniculatus & 61,20 & 52,15 & 50,48 & 75,30 & 75,80 & 75,45 \\
\hline L. uliginosus & 55,65 & $/ 1$ & $/ 1$ & 63,03 & $/ 1$ & $/ 1$ \\
\hline Média & 54,52 & 47,37 & 44,11 & 70,73 & 71,49 & 71,51 \\
\hline
\end{tabular}

Mean

${ }^{1}$ As espécies não atingiram os estádios fenológicos referidos.

${ }^{1}$ The species did not reach the stages related.

aérea, no início de florescimento (CAROSO et al., 1981). LÓPEZ et al. (1966) relataram DIVMS entre 65 e $81 \%$ em folhas e 58 e $68 \%$ em caules. OLIVEIRA e PAIM (1990) verificaram DIVMS de $51,77 \%$ no florescimento pleno. Segundo ROMERO (1990), os altos níveis de digestibilidade de cornichão estão associados aos baixos níveis de lignina. De qualquer maneira, a alta qualidade do cornichão através da estação de crescimento torna-o atrativo para pastejo durante o verão, quando a qualidade das gramíneas está baixa (ALISON e HOVELAND, 1989). Para $L$. uliginosus, CAROSO et al. (1982) encontraram valores de DIVMS entre 53 e $67 \%$ em estádio vegetativo. Por sua vez, SHILLER e AYRES (1994) observaram redução na DIVMO de 78 para 63\%, associada com alterações morfológicas induzidas pelas geadas, que reduziram significativamente a emissão de novas folhas. 


\section{Conclusões}

As leguminosas nativas A. latifolia, A. punctata e A. tristis detêm valores mínimos de PB para ruminantes, comparáveis aos de L. corniculatus e L. uliginosus. Entre as espécies de Adesmia estudadas, A. latifolia distingue-se das demais; sua dinâmica de acúmulo de biomassa aérea, teor de PB ehábito estolonífero estimulam a continuidade de estudos. Os resultados obtidos devem ser validados em condições de campo e, principalmente, em mistura com gramíneas ou sobre pastagem natural.

\section{Agradecimento}

Aos pesquisadores José Lino da Rosa, Nelson Eduardo Prestes (Epagri, SC) e Carlos Otávio Costa Moraes (Embrapa - CPPSul, RS), pelo fornecimento das sementes de Adesmia e Lotus.

\section{Referências Bibliográficas}

AKIN, D.E. 1989. Histological and physical factors affecting digestibility of forages. Agr. J., 81(1):17-25.

ALLEN, O.N., ALLEN, E.K. 1981. The Leguminosae. Madison: The University of Wisconsin Press. p.19-20.

BEUSELINCK, P.R., PETERS, E.J., McGRAW, R.L. 1984. Cultivar and management effects on stand persistence of birdsfoot trefoil. Agr. J., 76(2):490-492.

BICKOFF, E.M., KOTHER, G.O., SMITH, D. 1972. Chemical composition of herbage. In: HANSON, C.H. (Ed.). Alfalfa science and technology. Madison: American Society of Agronomy. p.247-282.

BULLARD, M.J., CRAWFORD, T.J. 1995. Productivity of Lotus corniculatus L. (birdsfoot trefoil) in the UK when grown under low-input conditions as spaced plants, monoculture swards or mixed swards. Grass For. Sci., 50(4):439-446.

BRINK, G.E., FAIRBROTHER, T.E. 1992. Forage quality and morphological components of diverse clovers during primary spring growth. Crop Sci., 21(4):1043-1048.

BURKART, A. 1952. Las leguminosas argentinas silvestres y cultivadas. Buenos Aires: ACME Agency. 569p.

CAROSO, G.F., PAIM, N.R., MARKUS, R. 1982. Avaliação de clones de Lotus uliginosus Schkuhr., em blocos de policruzamento. Pesq. Agrop. Bras., 17(4):617-622.

CAROSO, G.F., PAIM, N.R., PRATES, E.R. 1981. Avaliação da produção e persistência de progênies e cultivares de Lotus corniculatus L, Pesq. Agrop. Bras., 6(3):341-346.

CHAPMAN, D.F., CARADUS, J.R. 1997. Effects of improved, adapted whitecloverTrifoliumrepens L.) germplasmontheproductive properties of a hill pasture. N. Z. J. Agr. Res., 40(2):207-221.

COELHO, L.G.M. Citogenética e qualidade da forragem de espécies de Adesmia DC. nativas no Brasil. Santa Maria, RS: UFSM, 1996. 74p. Dissertação (Mestrado em Zootecnia), Universidade Federal de Santa Maria, 1996.

DALL' AGNOL, M., GOMES, K.E. Qualidade de forragem de acessos de gênero Adesmia. In: REUNIÃO ANUAL DA SOCIEDADE BRASILEIRA DE ZOOTECNIA, 31, 1994, Maringá. Anais... Maringá: SBZ, 1994. p.456.

DUELL, R.W., GAUSMAN, H.W. 1957. The effect of differential cutting on the yield, persistence, protein and mineral content of birdsfoot trefoil. Agr. J., 49(11):318-319.

DUTRA, G.M., MAIA, M. S., OLIVEIRA, J.C.P. Efeito de espaçamento e densidade de semeadura na produção de matéria seca de Adesmia latifolia no ano de estabelecimento. In: REUNIÃO DO GRUPO TÉCNICO EM FORRAGEIRAS DO CONE SUL - ZONA CAMPOS, 17, 1998, Lages. Anais... Lages: Epagri, 1998. p.123.

FORMOSO, F.A. 1993. Lotus corniculatus. I. Performance forrajera y caracteristicas agronómicas asociadas. Montevideo: INIA. 20p. (Boletin de divulgación, n.37).

HACKER, J.B., MINSON, D.J. 1981. The digestibility of plant parts. Herb. Abstr., 51(9):459-482.

HAYCOCK, R. 1984. Dry-matter distribution and seasonal yield changes in five contrasting genotypes of white clover. J. Agr. Sci., 102(2):333-340.

HILL JR., R.R., GUSS, S.B. 1976. Genetic variability for mineral concentration in plants related to mineral requirements of cattle. Crop Sci., 16(5):680-685.

HOPKINS, A., MARTYN, T. M., JOHNSON, R. H. et al. 1996. Forage production by two Lotus species as influenced by companion grass species. Grass For. Sci., 51(4):343-349.

LÓPEZ, J., PRESTES, P.J.Q., MAGALHÃES, E. A curva de crescimento e a composição em carboidratos solúveis, estruturais, lignina e proteína, e a digestibilidade em cornichão. In: CONGRESSO INTERNACIONAL DE PASTAGENS, 9., 1965, São Paulo. Anais... São Paulo: Alarico, 1966. p.851-857.

McGRAW, R.L., MARTEN, G.C. 1986. Analysis of primary spring growth of four pasture legume species. Agr. J., 78(4):704-710.

MIOTTO, S.T.S., LEITÃO FILHO, H.F. 1993. LeguminosaeFaboideae - Gênero Adesmia DC. Bol. Inst. Bioc., 53:1-157.

MORAES, C.O.C., DALL' AGNOL, M. 1990. Estado atual dos trabalhos de melhoramento de leguminosas de clima temperado. Bagé: EMBRAPA-CNPO. 22p. (Circular técnica, n.6)

MORAES, C.O.C., PAIM, N.R., NABINGER, C. 1989. Avaliação de leguminosas do gênero Trifolium. Pesq. Agrop. Bras., 24(7):813-818.

OLIVEIRA, J.C.P., PAIM, N.R. 1990. Teste de progênies em linha de seleção materna de duas espécies do gênero Lotus. Pesq. Agrop. Bras.,25(3):461-467.

PAIM, N. R. 1988. Research on Lotus spp. in Rio Grande do Sul, Southern Brazil. Lotus Newsletter, 19:37-43.

PAIM, N.R., RIBOLDI, J. Competição entre espécies e cultivares do gênero Lotus L. Pesq. Agrop. Bras., 26(10):1699-1704.

REIS, E.M.,FORCELINI, C.A. 1994. Manualdefungicidas: guia para ocontrole de doenças de plantas. Passo Fundo: Pe. Berthier. 100p.

RITTER, W., SORRENSON, W.J. 1985. Produção de bovinos no Planalto de Santa Catarina, Brasil - situação atual e perspectivas. Eschborn: GTZ. 172p.

ROMERO, Y. 1990. La lotera en la IX Region. Rev. Inv. Progr. Agropec., 9(3):28-30.

ROMO, J.T., EDDLEMAN, L.E. 1995. Use of degree-days in multiple-temperature experiments. J.R. Manag., 48(5):410-416.

SANTINAQUE, F., CARÁMBULA, M. 1981. Productividad y comportamiento de distintas mezclas forrajeras. Inv. Agr., 2(1):16-21.

SCHILLER, K.N., AYRES, J.F. 1993. The effects of winter conditions on the nutritive value of Lotus pedunculatus cv. Grasslands Maku na Trifolium repens cv. Haifa. Tr. Grassl., 27(1):43-47.

SOCIEDADE BRASILEIRA DE CIÊNCIA DO SOLO. Comissão de fertilidade do solo - RS/SC. Recomendações de adubação e de calagem para os Estados do Rio Grande do Sul e Santa Catarina. Santa Maria, 1997. 223p.

TILLEY, J.M.A., TERRY, R.A. 1963. A two-stage technique for the in vitro digestion of forage crops. J. Br. Grassl. Soc., 18:104-111.

VALLS, J.F.M. 1984. Notas sobre a taxonomia, disponibilidade de germoplasma e problemas para utilizaçãoforrageira de Adesmia spp. no sul do Brasil. Mat. dat., Porto Alegre. 11p.

Recebido em: 12/05/00 Aceito em: 31/04/01 